Pacific Journal of Mathematics

A POLYNOMIAL INVARIANT OF KNOTS AND LINKS 


\title{
A POLYNOMIAL INVARIANT OF KNOTS AND LINKS
}

\author{
Jim Hoste
}

\begin{abstract}
Suppose $L$ is a classical oriented link in $S^{3}$. We define an isotopy invariant of $L, Q_{L}(x, y, z)$, which is a homogeneous Laurent polynomial of degree zero. The Conway polynomial $\nabla_{L}(z)$ and the Jones polynomial $V_{L}(t)$ are special cases of $Q$. Some basic properties of $Q$ are given along with several examples.
\end{abstract}

1. Introduction. The definition of $Q$ is perhaps best conveyed by first recalling the iterative scheme by which the Conway polynomial (or reduced Alexander polynomial) of a knot or link can be computed starting from a projection of the link. (See for example [1], [4], or [6].) Suppose $P^{+}$, $P^{-}$, and $P^{s}$ are three oriented link projections which are identical except near a single crossing of $P^{+}$where they differ as shown in Figure 1.1.

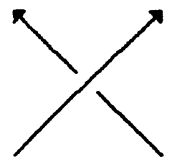

$p^{+}$

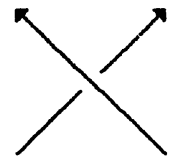

$P^{-}$

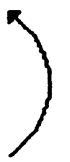

$p^{5}$

FIGURE 1.1

The projection $P^{-}$is obtained from $P^{+}$by changing the crossing and $P^{s}$ is obtained by smoothing the crossing. From the definition of the Conway polynomial $\nabla_{P}(z)$ it follows that

$$
\nabla_{P^{+}}(z)-\nabla_{P^{-}}(z)+z \nabla_{P^{s}}(z)=0 .
$$

Thus we may compute $\nabla_{L}(z)$ for a link $L$ by first choosing some projection $P$ of $L$ and then "resolving" $P$ into projections for which the Conway polynomial is known by repeatedly changing and smoothing crossings. We then make use of equation 1.1 to work backward to $P$. It also follows from the definition that $\nabla_{L}(z)=1$ if $L$ is the unknot and $\nabla_{L}(z)=0$ if $L$ is an unlink with two or more components. Hence it is always possible to compute $\nabla_{L}(z)$ this way by resolving $P$ into a collection of unlinks.

The recently discovered Jones polynomial, $V_{L}(t)$, can also be computed in this way [5]. However, instead of using equation (1.1), the following identity is used.

$$
t V_{P^{+}}(t)-t^{-1} V_{P^{-}}(t)+\left(t^{1 / 2}-t^{-1 / 2}\right) V_{P^{s}}(t)=0
$$


The "initial data" is also changed with an unlink of $k$ components now having the value $V_{L}(t)=(-(t+1) / \sqrt{t})^{k-1}$. Note that this is still equal to 1 if $L$ is the unknot.

These two invariants and the method by which they can be computed prompt the following definition.

Definition 1.1. Suppose $L$ is an oriented link. Let $P$ be any projection of $L$. Choose some resolution of $P$ into unlinks and then use this to compute $Q_{L}(x, y, z)$ as follows. First assign $Q$ values of $q_{k}=$ $(-(x+y) / z)^{k-1}$ to the unlinks having $k$ components. Next work backwards through the resolution using the identity

$$
x Q_{P^{+}}(x, y, z)+y Q_{P^{-}}(x, y, z)+z Q_{P^{s}}(x, y, z)=0
$$

to eventually assign a value of $Q$ to $P$. Call this $Q_{L}(x, y, z)$.

Notice that if $Q_{L}$ is to be an invariant of the link $L$ then we are nearly forced to choose $q_{k}=(-(x+y) / z)^{k-1}$. For consider the resolution illustrated in Figure 1.2.

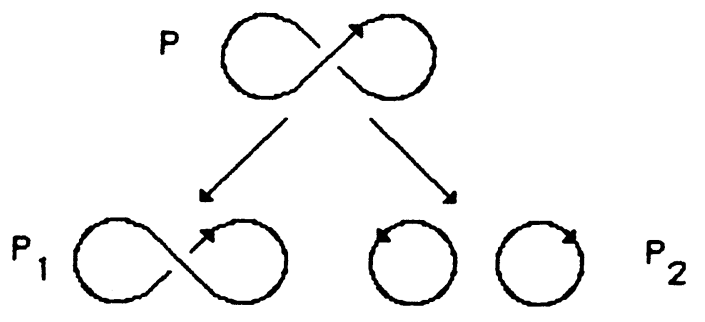

Figure 1.2

From equation (1.3) it follows that $q_{2}=Q_{P_{2}}=-((x+y) / z) q_{1}$, since $P_{1}$ and $P$ are both unknots. Similarly, one obtains $q_{k}=(-(x+y) / z)^{k-1} q_{1}$. Hence, once the value of the unknot polynomial has been chosen, the values of the unlinks are determined. Furthermore, the unknot polynomial will factor out of $Q_{L}$ for every $L$ and so we may normalize $Q_{L}$ by setting the value of $Q$ for the unknot equal to 1 .

Of course there are so many choices involved that there is no particular reason to believe that $Q$ is well defined. Nevertheless, we shall prove that this is indeed the case in $\$ 2$. The general plan of the proof is to first show that if $P$ is an oriented link projection then $Q_{P}$ is well defined, that is, independent of the choice of resolution. Once this is done it is relatively easy to show that $Q_{P}$ is preserved by Reidemeister moves and hence is an isotopy invariant of the link. However, the proof that $Q$ is well defined for projections is difficult, with the chief problem apparently 
arising from the fact that there is no canonical way to unlink a given projection by changing crossings. This difficulty is avoided by first giving an alternate definition of $Q$ for which the choice of resolution becomes almost canonical. The two definitions are later shown to be equivalent.

Section 2 will be devoted to showing that $Q$ is a well defined invariant of oriented isotopy type. Then in $\$ 3$ we enumerate a few basic properties of $Q$ and give several examples. Throughout this paper all links will be considered to be oriented and we shall work in the smooth category for convenience.

This research has as its origin the work of Vaughan Jones and his discovery of the trace invariant $V_{L}(t)$ [5]. The author attended a lecture given by Jones in the summer of 1984 and, upon seeing equation (1.2) and noting its similarity to equation (1.1), was led immediately to this investigation. Not surprisingly, so were several others. Different versions of $Q$ have been independently produced by Freyd and Yetter [2], Lickorish and Millett [7], Ocneanu [8], and Przytycki and Traczyk [9]. (See also [3].) The work of Lickorish and Millett and Przytycki and Traczyk is similar to that given here while the others' work is quite different.

2. The definition of $Q$. We will call a link projection ordered if the components have been given some ordering. A link projection is pointed if a point (different from a double point) has been distinguished on each component. In practice we will place a dot on each component at the distinguished point and label the components with integers according to their ordering.

An ordered link projection is layered with respect to its ordering if the $i$ th component never crosses beneath the $j$ th component if $i<j$. A link projection is layered if it is layered with respect to some choice of ordering.

A pointed ordered link projection is descending with respect to its ordering and pointing if it is layered with respect to its ordering and furthermore, at each crossing between strands of the same component the upper strand is reached before the lower strand if one travels along the knot in the same direction it is oriented having started at the distinguished point. A link projection is descending if it is descending with respect to some choice of ordering and pointing.

Notice that a descending link projection is an unlink projection.

Suppose $P$ is a link projection and that $P_{1}$ and $P_{2}$ are obtained from $P$ by respectively changing and smoothing some crossing. Then corresponding to these three projections is a graph having three vertices and two edges. The vertices correspond to the projections $P, P_{1}$, and $P_{2}$, and 
the edges join $P$ with $P_{1}$ and $P$ with $P_{2}$. This graph is called a tree of projections with root $P$. The projections $P_{1}$ and $P_{2}$ are outermost projections of the tree. The edge joining $P$ with $P_{1}$ is called a changing edge while the other edge is called a smoothing edge. We may build larger trees from smaller trees by changing and smoothing some crossing in an outermost projection, thus adding two more vertices and two more edges. In practice we will draw trees of projections with the root on top and the projections obtained from it below it. The changing edges will always be drawn going down and to the left, while the smoothing edges will be drawn going down and to the right. If all the smoothing (changing) edges of a tree are removed, then each connected component of what remains is called a changing (smoothing) branch. The changing (smoothing) branch that contains the root of the tree is called the initial changing (smoothing) branch. Notice that the number of crossings and the number of components in each projection along a given changing branch is constant.

When a crossing in a link projection is smoothed it may cause two different components to become one or a single component to become two. The former phenomenon is called a fusion while the latter is called a fission.

If the root of a tree is ordered then it induces orderings on all the other projections in the tree as follows. To begin with, if a crossing in an ordered projection is changed then the ordering of the components remains the same. If a fusion occurs between the $i$ th component and the $j$ th component, with $i<j$, then the resulting component is the new $i$ th component. All components numbered less than $j$ retain their labels while those numbered greater than $j$ have their labels reduced by 1 . If a fission occurs to the $i$ th component, then the two new components will be labeled $i$ and $i+1$ according to the following scheme. If one of the two components crosses over the other one more times than it crosses beneath it, then call it $i$ and the other one $i+1$. If this is not the case then label the right one $i$ and the left one $i+1$. The right one is the one that is on the right as one passes between the components in the same direction that they are oriented at the place where the smoothing occurred.

If the root of a tree is pointed in addition to being ordered then it also induces a pointing on each projection in the tree. This is done as follows. If a crossing is changed, the pointing is left unchanged. If a fusion occurs, the distinguished point on the lower numbered component is retained while the other distinguished point is discarded. If a fission occurs then a point is distinguished on the new component at the smoothing as indicated in Figure 2.1. 


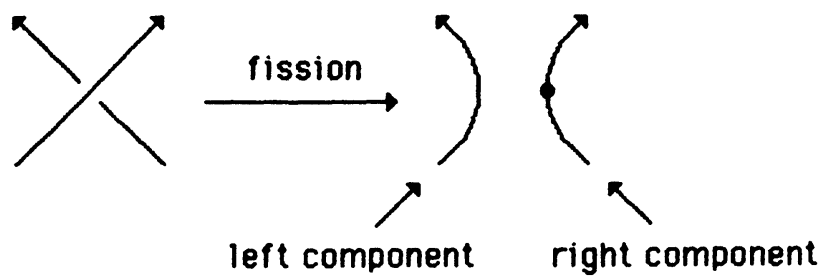

Figure 2.1

In this figure the left component retains the original distinguished point while the right component is assigned the new point.

If $P$ is a link projection then a resolution $R$ of $P$ is a tree $R$ having $P$ as root and such that each outermost projection of $R$ is an unlink projection.

We are now in a position to define $Q_{P}(x, y, z)$ for a link projection $P$. Suppose first that $P$ is a pointed ordered link projection, $R$ is a resolution of $P$, and finally that each outermost projection of $R$ is descending with respect to the ordering and pointing induced on it by $P$. We shall consider $R$ as a tree of pointed ordered link projections with each projection having the induced ordering and pointing. Such a resolution of a pointed ordered link projection is called a distinguished resolution. It is not hard to see that given any pointed ordered link projection $P$ there exists some distinguished resolution $R$ of $P$. Now assign to each outermost projection of $R$ having $k$ components the value $q_{k}=$ $(-(x+y) / z)^{k-1}$. We may now compute a value for each projection in $R$ by working backwards from the outermost projections and using equation (1.3).

$$
x Q_{P^{+}}(x, y, z)+y Q_{P^{-}}(x, y, z)+z Q_{P^{s}}(x, y, z)=0 .
$$

The value of $Q$ produced for the root of $R$ is called the value of the resolution and is denoted $Q(R)$.

Definition 2.1. Suppose $P$ is an oriented link projection. Choose some ordering and pointing of $P$ and then some distinguished resolution $R$ of $P$. Let $Q_{P}(x, y, z)=Q(R)$.

THEOREM 2.2. The Laurent polynomial $Q_{P}(x, y, z)$ is well defined.

Proof. We shall induct on the number of crossings in $P$. If there are no crossings then $P$ is descending with respect to every possible choice of ordering and pointing. Thus $Q(R)=q_{k}$ where $k$ is the number of 
components of $P$ for every choice of ordering, pointing, and distinguished resolution.

Now suppose that $P$ has $n$ crossings and that for any projection having fewer crossings $Q$ is well defined. The proof of the inductive step is broken into three major steps.

Step 1. Once the ordering and pointing of $P$ have been chosen, $Q$ is independent of the choice of distinguished resolution.

Proof of Step 1. Suppose that $R_{1}$ and $R_{2}$ are two distinguished resolutions of $P$ with respect to some chosen ordering and pointing of $P$. Now the initial changing branches of $R_{1}$ and $R_{2}$ must both end at the same projection. This is because the ordering and pointing of $P$ determine whether a crossing should be changed or not in order to make the projection descending.

Label the crossings of $P, c_{1}, c_{2}, \ldots, c_{n}$. Now the initial changing branch of every distinguished resolution $R$ of $P$ determines a word $w$ in the symbols $\left\{c_{1}^{ \pm 1}, c_{2}^{ \pm 1}, \ldots, c_{n}^{ \pm 1}\right\}$ as follows. On each changing edge in the initial changing branch of $R$ write $c_{i}$ if the $i$ th crossing was changed (from its original position in $P$ ) and $c_{i}^{-1}$ if it was changed back again (to its original position in $P$ ). Now we may read $w$ off of the initial changing branch starting at $P$ and ending at the outermost projection. Let $w_{i}$ be the word corresponding to $R_{i}$. Since the initial changing branches of both resolutions end at the same projection we see that the sum of the exponents of any $c_{i}$ is the same in $w_{1}$ as in $w_{2}$.

We will now prove the following two claims from which Step 1 follows easily.

Claim 1. If $w_{1}$ and $w_{2}$ differ by commuting two adjacent symbols (that correspond to different crossings) then $Q\left(R_{1}\right)=Q\left(R_{2}\right)$.

Claim 2. If $w_{1}$ and $w_{2}$ differ by the deletion of $c_{i} c_{i}^{-1}$ for some $i$ then $Q\left(R_{1}\right)=Q\left(R_{2}\right)$.

Proof of Claim 1. By using the inductive hypothesis we are free to replace any subtree of $R_{i}$ rooted at a projection $P^{\prime}$ having fewer than $\dot{n}$ crossings with another distinguished resolution of $P^{\prime}$ without changing $Q\left(R_{i}\right)$. Furthermore, we can even reorder and repoint $P^{\prime}$ and choose the new distinguished resolution subordinate to the new ordering and pointing. Thus we may assume that $R_{1}$ and $R_{2}$ are nearly the same. In 
particular let $P_{1}$ be the last projection where the two initial changing branches agree. Let $\bar{R}_{i}$ be the subtree of $R_{i}$ rooted at $P_{1}$. Then $\bar{R}_{1}$ and $\bar{R}_{2}$ are both distinguished resolutions of $P_{1}$ and it suffices to prove that $Q\left(\bar{R}_{1}\right)=Q\left(\bar{R}_{2}\right)$.

Suppose $\bar{R}_{1}$ begins by changing and smoothing $c_{i}$ in $P_{1}$. Let $P_{2}$ and $P_{4}$ be the respective resulting projections. If $c_{j}$ is next changed and smoothed in $P_{2}$, let $P_{3}$ and $P_{5}$ be the respective resulting projections. Now in $\bar{R}_{2}, c_{j}$ is changed first and then $c_{i}$ is changed. Let $P_{2}^{\prime}, P_{4}^{\prime}, P_{3}^{\prime}, P_{5}^{\prime}$, be the corresponding projections.

Since changing crossings does not affect ordering and pointing we see that $P_{3}=P_{3}^{\prime}$. Furthermore, the two initial changing branches are the same below $P_{3}$. Again, by using the inductive hypothesis, we may assume that the two subtrees of $\bar{R}_{1}$ and $\bar{R}_{2}$ rooted at $P_{3}$ and $P_{3}^{\prime}$ are the same. Call this subtree $T$.

The projections $P_{4}, P_{4}^{\prime}, P_{5}$, and $P_{5}^{\prime}$ all have $n-1$ crossings and so, by the inductive hypothesis, we are free to order, point, and resolve them in any way we wish without changing $Q\left(\bar{R}_{i}\right)$. So begin to resolve $P_{4}$ by changing and smoothing the $j$ th crossing. Similarly, begin to resolve $P_{4}^{\prime}$ by changing and smoothing the $i$ th crossing. The resolutions now appear, in part, as shown in Figure 2.2.

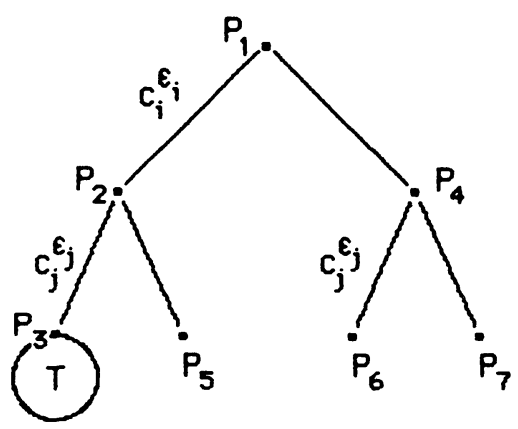

$\overline{R_{1}}$

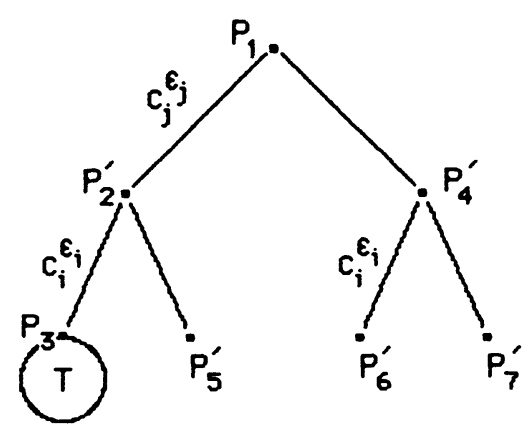

$\bar{R}_{2}$

FIGURE 2.2

Without regard to ordering and pointing it's clear that $P_{5}=P_{6}^{\prime}, P_{6}=$ $P_{5}^{\prime}$, and $P_{7}=P_{7}^{\prime}$. If we consider these projections with the ordering and pointing induced on them by $P_{1}$ then they may not be the same. But this does not matter.

Now there are four cases to be considered. The crossings $c_{i}$ and $c_{j}$ are either both right handed, both left handed, or mixed. Suppose they are 
both right handed. Then we have

$$
\begin{aligned}
Q\left(\bar{R}_{1}\right) & =x^{-2} y^{2} Q(T)+x^{-2} y z Q_{P_{5}}+x^{-2} y z Q_{P_{6}}+x^{-2} z^{2} Q_{P_{7}} \\
& =x^{-2} y^{2} Q(T)+x^{-2} y z Q_{P_{6}^{\prime}}+x^{-2} y z Q_{P_{5}^{\prime}}+x^{-2} z^{2} Q_{P_{7}^{\prime}} \\
& =Q\left(\bar{R}_{2}\right) .
\end{aligned}
$$

If they are both left handed, or mixed, then a similar calculation shows that $Q\left(\bar{R}_{1}\right)=Q\left(\bar{R}_{2}\right)$.

Proof of Claim 2. As in the proof of Claim 1 it suffices to prove that $Q\left(\bar{R}_{1}\right)=Q\left(\bar{R}_{2}\right)$ where $\bar{R}_{1}$ and $\bar{R}_{2}$ are shown in Figure 2.3 .

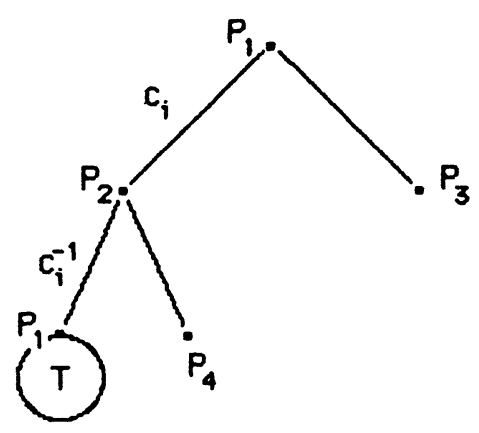

$\bar{R}$

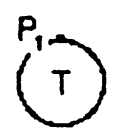

$\bar{R}_{2}=T$

Figure 2.3

Now $P_{3}$ is the same projection as $P_{4}$ even though the ordering and pointing induced on them may be different. If $c_{i}$ is a right handed crossing we obtain

$$
\begin{aligned}
Q\left(\bar{R}_{1}\right) & =Q(T)+x^{-1} z Q_{P_{4}}-x^{-1} z Q_{P_{3}} \\
& =Q(T)=Q\left(\bar{R}_{2}\right) .
\end{aligned}
$$

Starting out with a left handed crossing yields a similar calculation.

This completes the proof of Step 1 .

Step 2. Once the ordering of $P$ has been chosen, $Q$ is independent of the choice of pointing.

Proof. We will begin with the following special case. 
Claim 1. Suppose that $P$ is a pointed ordered link projection that is descending with respect to its ordering and pointing. Let $P^{\prime}$ be obtained from $P$ by moving one of the distinguished points forward along its component past one crossing. Then $Q_{P^{\prime}}=Q_{P}$.

Proof of Claim 1. There are two cases, each of which is illustrated in Figure 2.4.

Since $P$ is descending with respect to its ordering and pointing Case I can occur only if the overcrossing strand belongs to a different component. But now $P^{\prime}$ is descending with respect to its ordering and pointing. Thus $Q_{P^{\prime}}=Q_{P}$.

In Case II, if the undercrossing strand belongs to another component then again, $P^{\prime}$ is descending with respect to its ordering and pointing.

So suppose that both strands belong to the same component. The right handed case is illustrated in Figure 2.5.

Since $P^{\prime}$ is not descending we resolve it by changing and smoothing the crossing shown in Figure 2.5. Now $P_{1}$ is descending with respect to its induced ordering and pointing since $P$ was. Furthermore, so is $P_{2}$ ! Now

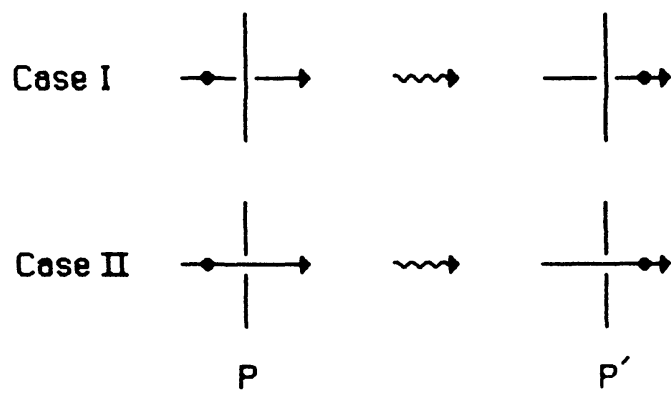

FIGURE 2.4
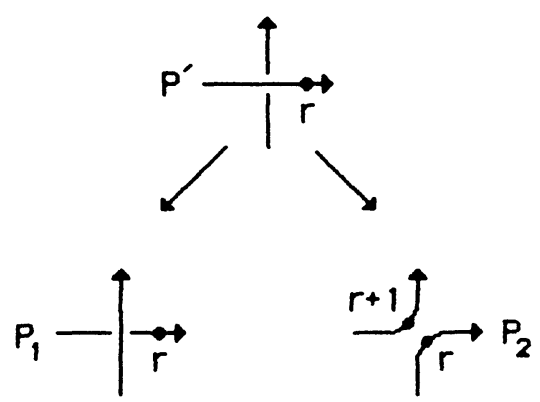

FIGURE 2.5 
by Step 1 we may use this resolution to compute $Q_{P^{\prime}}$. We get

$$
Q_{P^{\prime}}=-x^{-1} y q_{k}-x^{-1} z q_{k+1}=q_{k}=Q_{P} .
$$

The left handed case is similar.

Now suppose that $P$ is a pointed ordered link projection and that $P^{\prime}$ is obtained from it by repointing it. We wish to prove that $Q_{P^{\prime}}=Q_{P}$. It suffices to consider the case where $P^{\prime}$ is obtained by simply moving one distinguished point forward past one crossing. Let $R$ be a distinguished resolution of $P$ and consider the initial changing branch of $R$ together with all the projections immediately descendent from the initial changing branch. Let $T$ be this subtree of $R$. Call the projection at the end of the initial changing branch $P_{m}$ and the other outermost projections of $T, P_{1}$ through $P_{m-1}$. Let $T^{\prime}$ be obtained from $T$ by repointing the root as $P^{\prime}$ and inducing new pointings on the rest of $T$. Call $P_{i}^{\prime}$ the corresponding outermost projections of $T^{\prime}$. Now $P_{m}$ and $P_{m}^{\prime}$ satisfy the hypothesis of Claim 1 and so $Q_{P_{m}^{\prime}}=Q_{P_{m}}$. Furthermore, without regard to ordering and pointing, $P_{i}^{\prime}=P_{i}$ for $i<m$. Hence, by our inductive hypothesis, $Q_{P_{i}^{\prime}}$ and $Q_{P_{i}}$ are both well defined and equal. Thus $Q_{P^{\prime}}=Q_{P}$.

Step 3. $Q_{P}$ is independent of the choice of ordering.

Proof. We begin with the following reduction.

Claim 1. It suffices to prove Step 3 in the case where $P$ is descending with respect to some choice of ordering and pointing.

Proof of Claim 1. Let $P$ be given some ordering and let $P^{\prime}$ be $P$ endowed with some other ordering. Choose some pointing for $P$ and some distinguished resolution $R$. Again, let $T$ be the subtree of $R$ consisting of the initial changing branch together with all the immediately descendent projections. Reorder the root of $T$ as $P^{\prime}$ and induce new orderings and pointings on $T$ to obtain a new tree called $T^{\prime}$. Without regard to their ordering and pointing we have again that $P_{i}^{\prime}=P_{i}$ for $i<m$. Since these projections have $n-1$ crossings it follows that their polynomials are well defined and equal. Hence it only remains to prove that $Q_{P_{m}^{\prime}}=Q_{P_{m}}$.

But $P_{m}$ is descending and so we need only prove Step 3 for descending projections.

Claim 2. It suffices to prove Step 3 in the case where $P$ is descending and has two components. 
Proof of Claim 2. Suppose $P$ is a descending projection with $k$ components that has been given some ordering. Since $P$ is layered we may read the labels off the components starting at the "top" and working down. This gives a $k$-tuple $\left(i_{1}, i_{2}, \ldots, i_{k}\right)$. Suppose the ordering is not the preferred one, that is, the one with $i_{j}<i_{j+1}$ for all $j$. Then it may be restored to the preferred one by repeatedly transposing a pair of consecutive labels that are out of order. This can be done in a canonical way by first moving 1 to the front, then 2 to the front, etc. We shall induct on the number of such transpositions needed to restore the order to the preferred order.

If none are needed then $Q_{P}=q_{k}$ since $P$ is layered with respect to its ordering.

Now suppose that $P$ requires $m$ such transpositions. Let $P^{\prime}$ denote the two component sublink of $P$ to which the first transposition takes place. In other words, $P^{\prime}$ consists of the $i_{j}$ and $i_{j+1}$ components of $P$ with $i_{j}>i_{j+1}$ and $i_{j+1}$ is the smallest label which is out of order. So $P^{\prime}$ is not layered with respect to its ordering even though it is descending with respect to some choice of ordering and pointing. Give $P^{\prime}$ that choice of pointing and let $R^{\prime}$ be a distinguished resolution of $P^{\prime}$. The effect of the initial changing branch of $R^{\prime}$ is to relayer the components of $P^{\prime}$. Now in each projection of $R^{\prime}$ insert the remaining components of $P$ to obtain a tree $R$. Let $P_{2}$ be an outermost projection of $R$ and $P_{2}^{\prime}$ the corresponding projection of $R^{\prime}$. We will show that $Q_{P_{2}}=q_{k-1} Q_{P_{2}^{\prime}}$. This implies that $Q_{P}=q_{k-1} Q\left(R^{\prime}\right)$. Thus it suffices to prove Step 3 for a two component descending projection. We could then conclude that $Q\left(R^{\prime}\right)=q_{2}$ and hence that $Q_{P}=q_{k}$.

To finish the proof, assume first that $P_{2}$ is at the end of the initial changing branch of $R$. Then the order of the components of $P_{2}$ can be restored in $m-1$ transpositions and so, by our inductive hypothesis, $Q_{P_{2}}=q_{k}=q_{k-1} q_{2}=q_{k-1} Q_{P_{2}^{\prime}}$. If $P_{2}$ is not at the end of the initial changing branch then $P_{2}$ has less than $n$ crossings. But $P_{2}$ is descending since $P_{2}^{\prime}$ is and $P_{2}$ is obtained from $P_{2}^{\prime}$ by inserting $k-2$ more descending components. Thus $Q_{P_{2}}=q_{k-2+r}=q_{k-1} q_{r}=q_{k-1} Q_{P_{2}^{\prime}}$, where $r$ is the number of components of $P_{2}^{\prime}$.

Now to finish the proof of Step 3 we will prove the following two claims.

Claim 3. If $P$ is a descending projection with two components and any number of crossings, then $P$ can be transformed into a split projection (i.e. one with no crossings between the components) by a finite 
number of generalized Reidemeister moves. By a generalized Reidemeister move we mean the moves shown below where the shaded region may be unbounded (i.e. contain the point at infinity if the projection is regarded as lying in $\left.S^{2}=\mathbf{R}^{2} \cup\{\infty\}\right)$.

I

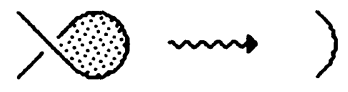

II

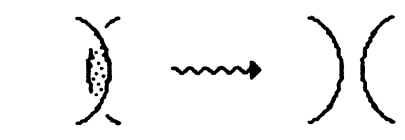

III
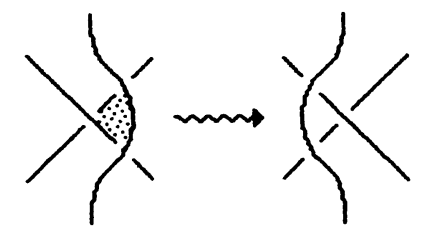

or

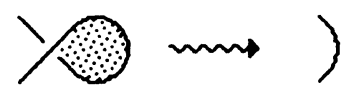

or

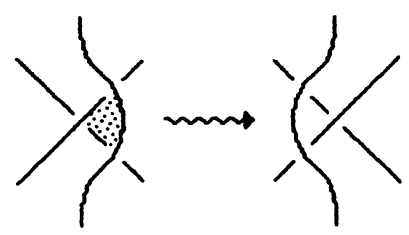

Note that we do not allow the inverses of these moves. Furthermore, notice that after a move of this type is applied to $P$, the projection remains descending with two components, although the number of crossings may decrease.

By a 1-gon, 2-gon or 3-gon, we mean a region of the complement of the projection like the shaded regions shown above.

Claim 4. Let $P$ be a descending ordered link projection with two components and $n$ or less crossings. ( $P$ may not be layered with respect to its given ordering.) If $P$ is changed by a generalized Reidemeister move then $Q_{P}$ is preserved.

Once these last two claims are proven we may finish the proof of Step 3 as follows. Suppose $P$ is an ordered two component projection with $n$ crossings that is descending with respect to some choice of ordering and pointing. By Claim 3 we may transform $P$ into a split descending projection $P^{\prime}$ by a finite sequence of generalized Reidemeister moves. Each projection in this sequence satisfies the hypothesis of Claim 4 and hence $Q_{P}=Q_{P^{\prime}}$. But $Q_{P^{\prime}}$ is independent of ordering and equals $q_{2}$ since $P^{\prime}$ is layered with respect to any choice of ordering.

Proof of Claim 3. Suppose $P$ is a descending projection with two components, $K$ and $J$, where $K$ is the upper component. We may assume $J$ crosses beneath $K$, for if not, $P$ is already split. We shall induct on the number of crossings in the projection of $K$ alone. 


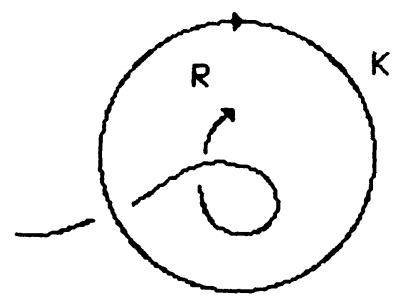

Figure 2.6

Suppose first that the projection of $K$ alone has no crossings. Now $K$ divides the plane into two regions. Let $R$ be the region that does not contain the distinguished point of $J$. Strands of $J$ enter $R$, travel around inside $R$, and then leave $R$. Suppose a strand of $J$ crosses itself inside $R$ before leaving. This strand forms a 1-gon as shown in Figure 2.6.

If no other strands of $J$ cross this 1-gon then it can be eliminated via a generalized Reidemeister move of type I. (Note that the 1-gon might contain the point at infinity. For the sake of convenience we shall draw all the remaining figures in this step as projections in $\mathbf{R}^{2} \cup\{\infty\}$.) So assume strands of $J$ across this 1-gon. Proceed in this manner until an "innermost" 1-gon is found, that is, a 1-gon in which, if strands do enter they leave without crossing themselves. Now if this 1-gon is empty, eliminate it. If not, then a strand which enters and leaves it forms a 2-gon. We can be sure of this since the distinguished point of $J$ lies outside $R$. The projection now looks, in part, like Figure 2.7.

Now if the 2-gon is empty, eliminate it. If strands enter it they do not cross themselves inside since we are already inside an innermost 1-gon. If strands enter and exit on the same side, or if two strands cross each other inside then there exists a "smaller" 2-gon. Again, it is important to note that the distinguished point of $J$ lies outside $R$. So finally there is a 2-gon with no smaller 2-gon inside. Strands that cross it enter on one side and leave on the other and cross another strand inside it at most once. Thus the strands that cross the 2-gon form a braid as shown in Figure 2.8. The figure is drawn only with double points.

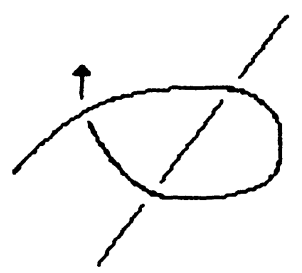

or

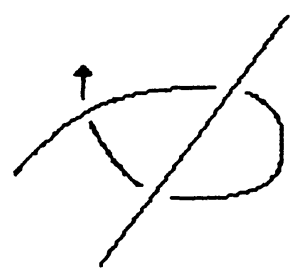

FIGURE 2.7 


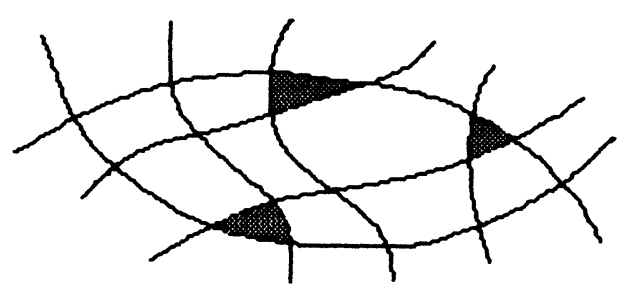

FIGURE 2.8

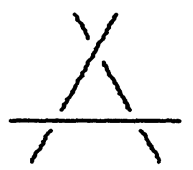

a

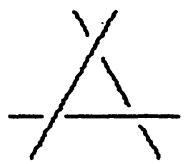

e

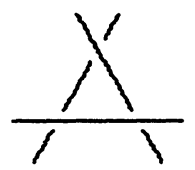

b

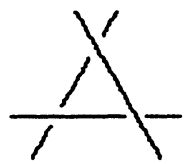

f

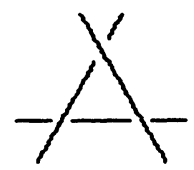

C

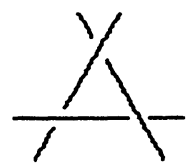

$\mathbf{g}$

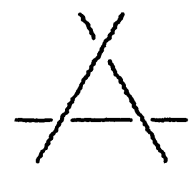

d

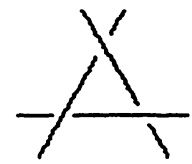

h

FIGURE 2.9

Now there must exist at least one triangle as shaded in Figure 2.8 at each side of the 2-gon. The strands of the triangle must appear as in cases (a)-(f), in Figure 2.9.

Cases (g) and (h) are impossible since $J$ is descending and, again, the distinguished point of $J$ lies outside of $R$. Thus a move of type III will move one crossing out of the 2-gon. Eventually the strands crossing the 2-gon will do so without crossing each other. Now moves of type III can be used to clear the 2-gon. Finally, the 2-gon can be eliminated using a move of type II.

Repeatedly doing this allows us to ultimately clear the 1-gon and then eliminate it. So eventually we may arrive at the situation where strands of $J$ that enter $R$ leave without crossing themselves. If two such strands form a 2-gon then the above argument may be repeated to eliminate it. Hence we may assume that strands of $J$ cross $R$ without self crossings and no two strands have more than one crossing between them. Now a strand of $J$ that crosses $R$ forms a 2-gon with $K$ and we may suppose that this 2-gon is "innermost". Again clear it by using moves of type III and then eliminate it by using a move of type II. Eventually we may clear $R$ and thus arrive at a split projection. 
Notice that the above argument actually proves the following. If a 1 -gon is formed by some strand of $K$ or $J$ (or a 2-gon by two strands of $K$ or two strands of $J$ ) such that the 1-gon (or 2-gon) does not contain either distinguished point in its interior or on its boundary then by using a finite number of generalized Reidemeister moves we may move any strands of $K$ or $J$ out of the 1-gon (or 2-gon). Moreover, it is not hard to prove the above statement for 3-gons. We leave this as an exercise for the reader.

Now suppose that the projection of $K$ alone has $n$ crossings. To prove the inductive step we consider the following classes of "arc projections". Let $K_{i}$ be the collection of all descending projections of an oriented arc in $\mathbf{R}^{2} \cup\{\infty\}$ which have $i$ crossings. We consider these projections only up to an equivalence which should soon be clear to the reader. Figure 2.10 illustrates $K_{1}$ and $K_{2}$.
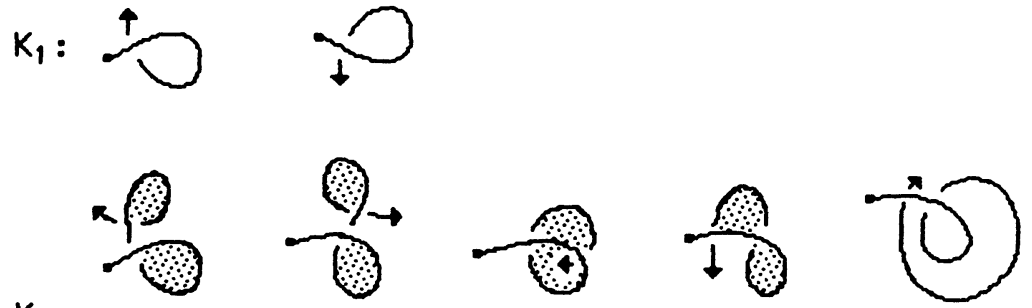

$K_{2}$ :
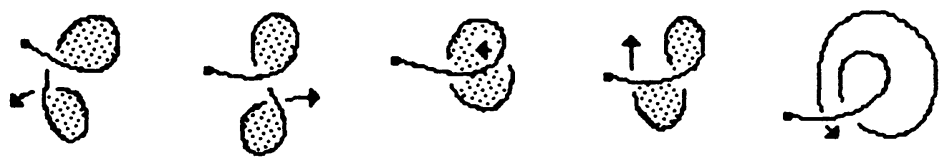

FIGURE 2.10

Notice that each projection in $K_{i}$ can be obtained from a projection in $K_{i-1}$ by extending the arc and introducing one more crossing. Furthermore, each descending knot projection with $i$ crossings can be obtained by "closing" some projection in $K_{i}$, that is, by extending the arc to form a closed curve without introducing any more crossings.

Now if $\alpha$ is a projection of $K_{i}$ we call $\alpha$ good if either of the following conditions hold.

1. The complement of $\alpha$ either contains two 1-gons, or one 1-gon and one 2-gon, neither of which contain the initial point of $\alpha$ in their interior, or $\alpha$ is the extension of such a projection.

2. There exists a finite sequence of arc projections $\alpha_{1}, \alpha_{2}, \ldots, \alpha_{m}$ satisfying the following three conditions.

(a) $\alpha$ is an extension of $\alpha_{1}$. 
(b) The complement of each $\alpha_{i}$ contains a 1-gon and a 3-gon neither of which contain the initial point of $\alpha_{i}$ in their interior and such that a move of type III can be applied to the 3-gon to obtain an extension of $\alpha_{i+1}$.

(c) $\alpha_{m}$ satisfies condition 1 .

A projection in $K_{i}$ is called bad if it is not good. So each projection of $K_{1}$ is bad while only two projections of $K_{2}$ are bad. Clearly each bad projection in $K_{i}$ must be the extension of a bad projection in $K_{i-1}$. Thus it is not hard to prove by induction on $i$ that each bad projection in $K_{i}$ must appear as one of the projections in Figure 2.11.
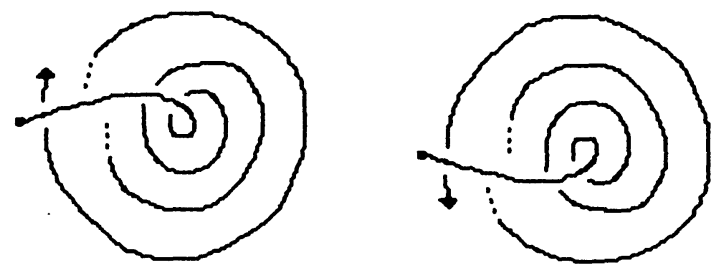

FIGURE 2.11

For suppose $\alpha \in K_{i}$ is a bad projection. Then $\alpha$ is the extension of a bad projection $\beta \in K_{i-1}$. Now by assumption $\beta$ appears as in Figure 2.11. But now it is easy to check that all possible extensions of $\beta$ are either good or appear as claimed.

Finally, we return to the proof of the inductive step. Suppose $K$ has $n$ crossings and is the closure of $\alpha \in K_{n}$. If $\alpha$ is good and condition 1 is true then the distinguished point of $J$ does not lie in one of the 1 or 2-gons. Hence it may be cleared and the number of crossings in $K$ reduced. If $\alpha$ is good and condition 2 holds then we may either clear the 1-gon and then reduce the number of crossings of $K$ or clear the 3-gon and repeat until eventually we reduce the number of crossings of $K$.

Finally, if $\alpha$ is bad then closing $\alpha$ to form $K$ yields a projection with two 1-gons in its complement. One of these may be cleared and eliminated to reduce the number of crossings of $K$.

Proof of Claim 4. Let $P$ be an ordered two component link with $n$ or less crossings that is descending with respect to some choice of ordering and pointing. Suppose first that $P^{\prime}$ is obtained from $P$ by a Reidemeister move of type I. Let $c$ be the crossing that is eliminated by the move. Give $P$ its preferred pointing. Since each component of $P$ is descending with respect to its pointing we may begin to resolve $P$ without changing $c$ along the initial changing branch. The effect of the initial changing branch is to relayer the components. Each projection obtained by smoothing a 
crossing is a knot still containing $c$ and the 1-gon that is to be eliminated by the Reidemeister move. Since these knot projections have $n-1$ crossings we are free to repoint them and resolve them however we wish. Thus we may begin to resolve them in such a way that we again avoid changing $c$. Continuing in this way we may build a resolution $R$ for $P$ in which $c$ is never changed and each projection contains the 1-gon that is to be eliminated just as it appeared in $P$.

Now create a new tree $R^{\prime}$ with $P^{\prime}$ as root by performing the Reidemeister move to each projection of $R$. Give $P^{\prime}$ the ordering and pointing it inherits from $P$ and use it to induce orderings and pointings on all of $R^{\prime}$. Now the outermost projections of $R^{\prime}$ are descending since those of $R$ are and the Reidemeister moves preserve this quality. But the only outermost projections of $R^{\prime}$ that might not be descending with respect to their induced ordering and pointing are those with less than $n$ crossings. Thus $Q\left(R^{\prime}\right)=Q(R)$ and so $Q_{P^{\prime}}=Q_{P}$.

Now suppose $P^{\prime}$ is obtained from $P$ by a Reidemeister move of type II. Suppose first that the two strands of $P$ involved in the move either belong to the same component of $P$, or if not, that the higher strand belongs to the first component. Then as before, $P$ admits a resolution $R$ in which these two crossings are never changed. Perform the move to each projection of $R$ to obtain a new tree $R^{\prime}$ with $P^{\prime}$ as root. Again $Q\left(R^{\prime}\right)=$ $Q(R)$ and so $Q_{P^{\prime}}=Q_{P}$.

If the two strands belong to different components and the first component is lower, consider the tree shown in Figure 2.12.

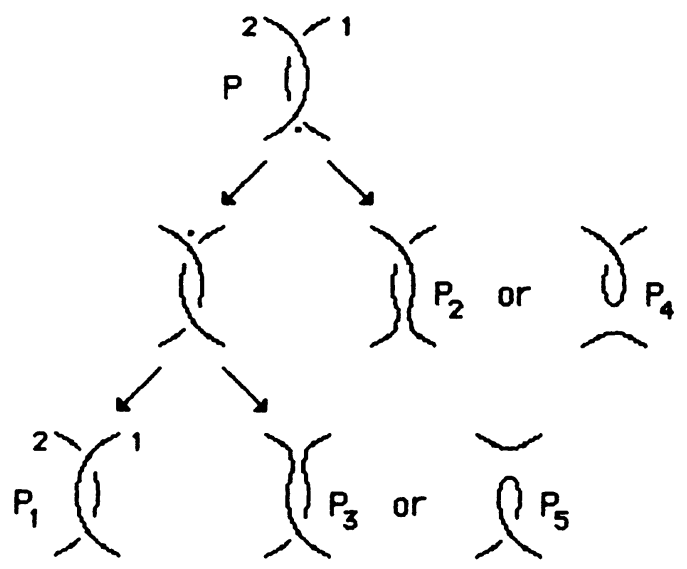

FIGURE 2.12 


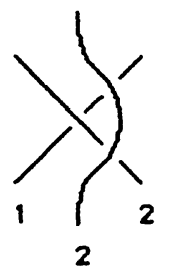

o

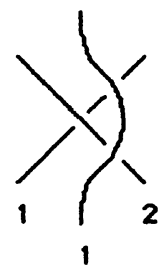

b

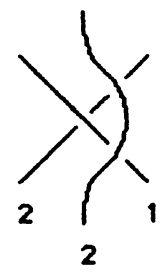

C

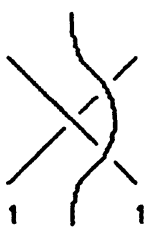

2

d

FIGURE 2.13

Depending on how the strands are oriented we obtain $P_{2}$ and $P_{3}$, or $P_{4}$ and $P_{5}$. Now clearly $Q_{P_{2}}=Q_{P_{3}}$ and furthermore $Q_{P_{4}}=Q_{P_{5}}$ since $P_{4}$ and $P_{5}$ can each be reduced to the same projection by a Reidemeister move of type I. It will then follow that $Q_{P}=Q_{P_{1}}$. But $Q_{P_{1}}=Q_{P^{\prime}}$, since the strands are now correctly layered.

Finally, consider a move of type III. Again, consider first the case where the three strands belong to the same component, or if not, are at least layered correctly. Then we may construct a resolution $R$ of $P$ never changing the three crossings in question. Again let $R^{\prime}$ result by performing the move in each projection of $R$. Once again we have $Q\left(R^{\prime}\right)=Q(R)$ and so $Q_{P^{\prime}}=Q_{P}$.

Now suppose the three strands belong to two components and are mislayered. There are four possible cases as shown in Figure 2.13.

Consider case (a) first and the two trees shown in Figure 2.14. As in the case of a move of type II, the smoothings cannot be specified without knowing the orientation of the strands. However, the smoothing appears the same in $P_{i}$ as in $P_{i}^{\prime}$ for $i=2,3$. Thus, $Q_{P_{i}}=Q_{P_{1}^{\prime}}$ for $i=2,3$. Furthermore, $Q_{P_{i}^{\prime}}=Q_{P_{1}}$ since $P_{1}$ and $P_{1}^{\prime}$ differ by a Reidemeister move of type III where the strands are correctly layered. It now follows that $Q_{P}=Q_{P}$, regardless of how the strands are oriented.

Cases (b), (c), and (d) are similar and are left to the reader.

This completes the proof of Step 3 and of Theorem 2.2.

Definition 2.3. Suppose $L$ is an oriented link in $S^{3}$. Let $P$ be any projection of $L$ and define $Q_{L}(x, y, z)=Q_{P}(x, y, z)$.

TheOREM 2.4. The Laurent polynomial $Q_{L}(x, y, z)$ is well defined and depends only on the isotopy class of $L$.

COROLlary 2.5. If $L$ is an unlink of $k$ components then $Q_{L}(x, y, z)=$ $(-(x+y) / z)^{k-1}$. 


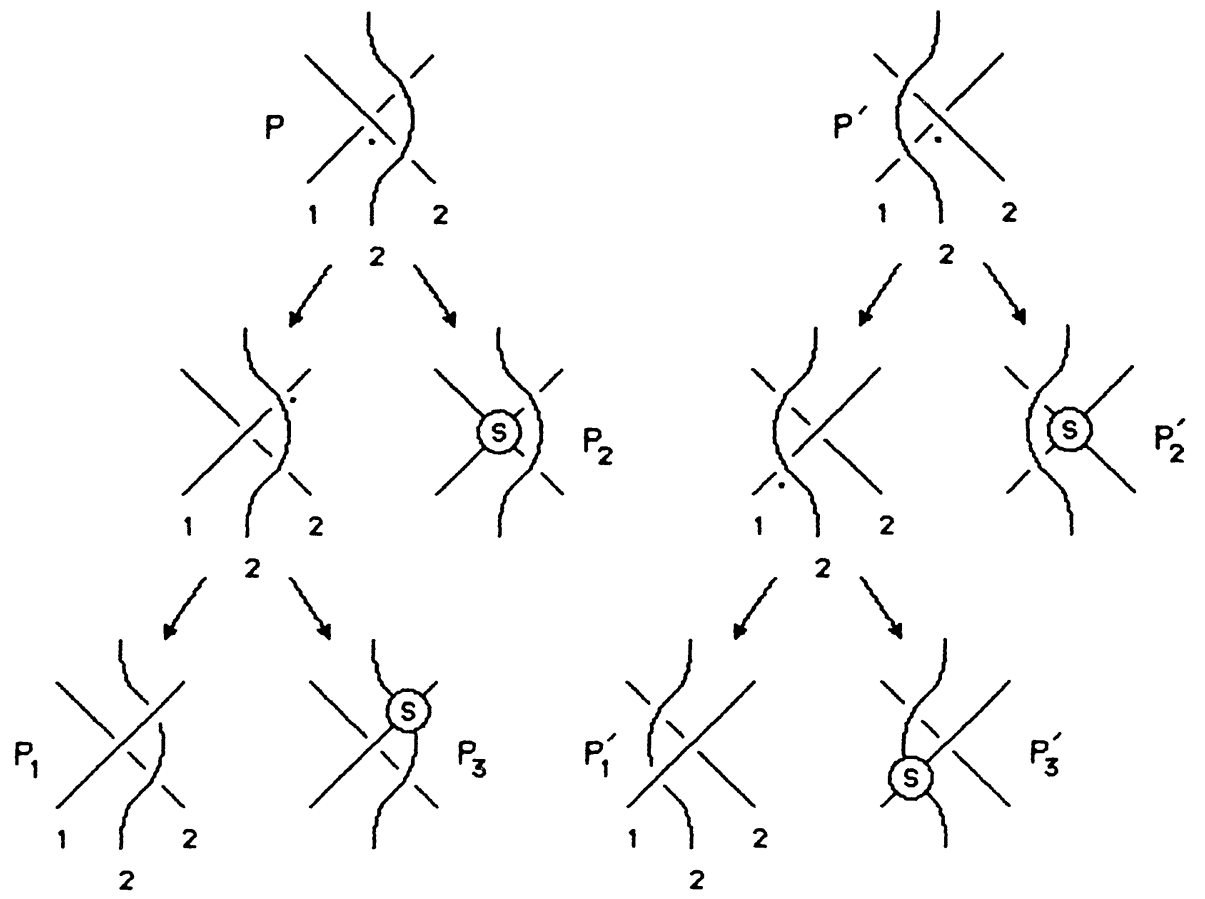

FIGURE 2.14

Proof of 2.4. Suppose $P_{1}$ and $P_{2}$ are two projections of $L$. Since $P_{1}$ can be transformed into $P_{2}$ by a finite sequence of Reidemeister moves, and their inverses, it suffices to prove that $Q_{P}$ is preserved by such moves. Since we now know that $Q_{P}$ is independent of the choice of pointing, ordering, and resolution, we may proceed as we did in Claim 4 of Step 3 of the proof of Theorem 2.2. Namely, we may find a resolution $R$ of $P$ in which the crossings involved in the Reidemeister move are never changed. Now perform the move in each projection of $R$ to obtain a new resolution $R^{\prime}$. The root $P^{\prime}$ of $R^{\prime}$ differs from $P$ by the Reidemeister move and furthermore $Q\left(R^{\prime}\right)=Q(R)$ so that $Q_{P^{\prime}}=Q_{P}$.

Corollary 2.5 is obvious since if $L$ is the unlink we may now consider for $P$ the trivial projection.

Theorem 2.6. Definition 1.1 produces the same polynomial as Definitions 2.1 and 2.3.

Proof. Choose some projection $P$ of $L$ and suppose that $R$ is a resolution of $P$ with respect to Definition 1.1. In other words, $R$ is a tree with $P$ as root and every outermost projection of $R$ is an unlink 
projection. Now order and point $P$ and induce orderings and pointings on all of the projections of $R$. With respect to this choice, $R$ may not be a distinguished resolution. However, we may extend $R$ to a distinguished resolution $R^{\prime}$ by further resolving its outermost projections if necessary. But by Corollary 2.5 the outermost projections of $R$ have well defined $Q$ values of $q_{k}$, where $k$ equals the number of components. Hence $Q\left(R^{\prime}\right)=$ $Q(R)$ and the definitions agree.

3. Properties of $Q_{L}(x, y, z)$. In this section we will describe some basic properties of $Q$. The first two properties follow immediately from the definition.

1. $Q_{L}(x, y, z)$ is a homogeneous Laurent polynomial of degree zero.

2. The Alexander, Conway, and Jones polynomials may be recovered from $Q$ by the following formulae:

$$
\begin{aligned}
\Delta_{L}(t) & =Q_{L}\left(1,-1, t^{1 / 2}-t^{-1 / 2}\right) \\
\nabla_{L}(z) & =Q_{L}(1,-1, z) \\
V_{L}(t) & =Q_{L}\left(t,-t^{-1}, t^{1 / 2}-t^{-1 / 2}\right) .
\end{aligned}
$$

3. If the orientations of all the components of $L$ are reversed then $Q_{L}$ remains unchanged.

This is clear since the same resolution may be used to compute $Q_{L}$ both before and after the orientations are reversed.

4. If $r L$ is the mirror image of $L$ (gotten by changing all the crossings in some projection of $L)$ then $Q_{r L}(x, y, z)=Q_{L}(y, x, z)$.

To see this, suppose $P$ is a projection of $L$ and $R$ a resolution of $P$. Let $r R$ be obtained from $R$ by changing all the crossings in each projection of $R$. Then $r R$ is a resolution for $r P$, a projection of $r L$. But changing and smoothing a crossing in $R$ corresponds to changing and smoothing a crossing of the opposite handedness in $r R$.

We may restate Property 4 as,

5. If the orientation of $S^{3}$ is reversed then $Q_{L}(x, y, z)$ becomes $Q_{L}(y, x, z)$.

6. If $L$ is amphicheiral, that is, isotopic to $r L$, then $Q_{L}(x, y, z)=$ $Q_{L}(y, x, z)$.

7. Suppose that $L$ is split. In other words, $L=L_{1} \cup L_{2}$ and there is a 2-sphere in $S^{3}-L$ separating $L_{1}$ from $L_{2}$. Then $Q_{L}=q_{2} Q_{L_{1}} Q_{L_{2}}$.

Proof. Let $P_{1}, P_{2}, R_{1}$ and $R_{2}$ be projections and resolutions respectively of $L_{1}$ and $L_{2}$. We may build a resolution $R$ for $L$ as follows. First insert $P_{2}$ in each projection of $R_{1}$ to create a tree $S$ with $L$ as root. Each 
outermost projection of $S$ consists of $P_{2}$ together with some unlink projection. Hence we may further resolve the outermost projections of $S$ by using $R_{2}$ and carrying the unlink projections along through $R_{2}$. Now it is not difficult to show that $R$ produces the polynomial $Q(R)=$ $q_{2} Q_{L_{1}} Q_{L_{2}}$.

We may define a connected sum $L_{1} \# L_{2}$ of two links $L_{1}$ and $L_{2}$ as follows. First place $L_{1}$ and $L_{2}$ in separate hemispheres of $S^{3}$. Next, form the ordinary connected sum of some component of $L_{1}$ with some component of $L_{2}$ using a band which meets the equator of $S^{3}$ exactly once.

8. Suppose $L=L_{1} \# L_{2}$. Then $Q_{L}=Q_{L_{1}} Q_{L_{2}}$.

Proof. From Figure 3.1 and Property 7 it follows that

$$
x Q_{L}+y Q_{L}=-z q_{2} Q_{L_{1}} Q_{2}=(x+y) Q_{L_{1}} Q_{L_{2}}
$$

so that $Q_{L}=Q_{L_{1}} Q_{L_{2}}$.

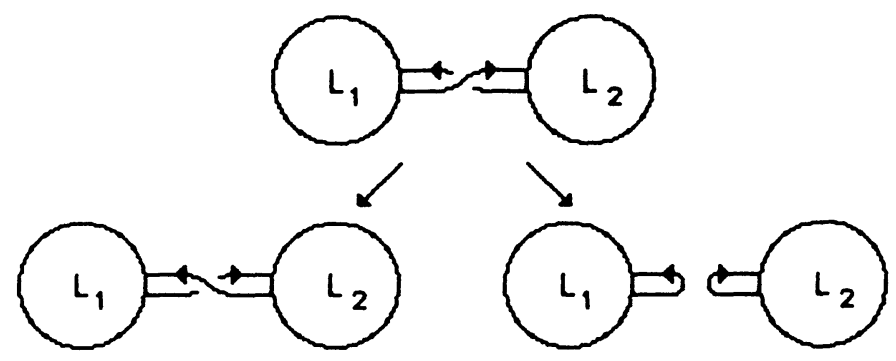

FIGURE 3.1

Using Property 8 and the fact that the connected sum of two links is not well defined, one can easily create distinct links having the same polynomial. However, we will soon show that there exist distinct prime knots having the same polynomial.

9. Suppose $L$ has $k$ components. Then we may write $Q_{L}$ as $Q_{L}=$ $z^{1-k} Q_{L}^{\prime}$ where $Q_{L}^{\prime}$ has only non-negative even powers of $z$ and furthermore $Q_{L}^{\prime}(x, y, 0) \neq 0$. Thus $Q_{L}$ detects the number of components of $L$ and also is never zero.

Proof. Let $m$ be the minimum number of crossings in any projection of $L$. We shall induct on $m$. If $m=0$ then $L$ is the unlink and $Q_{L}=q_{k}$ which has the desired form.

Now suppose $L$ has a projection $P$ with $m$ crossings but that any link having a projection with fewer crossings has a polynomial as described above. We now consider two cases. Suppose first that $L$ has one component. Let $R$ be a resolution of $P$ and let $T$ be the subtree of $R$ which consists of the initial changing branch of $R$ together with the 
immediately descendent projections. Label the outermost projections of $T, P_{1}$ through $P_{n}$, with $P_{n}$ at the end of the initial changing branch. Now each $P_{i}$, for $i \leq n-1$, has two components and less than $m$ crossings. Hence each $Q_{P_{i}}=z^{-1} Q_{P_{i}}^{\prime}$ has only non-negative even powers of $z$ and furthermore $Q_{P_{i}}^{\prime}(x, y, 0) \neq 0$. Also, $P_{n}$ is an unknot and so $Q_{P_{n}}=1$. Therefore we have,

$$
Q_{P}=a_{n}+\sum a_{i}\left(-z / b_{i}\right) Q_{P_{i}}=a_{n}-\sum a_{i} b_{i}^{-1} Q_{P_{t}}^{\prime}
$$

where $b_{i}$ is either $x$ or $y$ and $a_{i}=(-y / x)^{r_{i}}(-x / y)^{l_{i}}$ with $r_{i}$ and $l_{i}$ being the number of right and left handed crossings changed to reach $P_{i}$. Since each $Q_{P_{i}}^{\prime}$ has only non-negative even powers of $z$ it follows that this is true for $Q_{P}=Q_{P}^{\prime}$ as well. Furthermore, since $L$ is a knot we have $\nabla_{L}(0)=1$. Hence $Q_{P}^{\prime}(1,-1,0)=1$ and so $Q_{P}^{\prime}(x, y, 0) \neq 0$.

Now suppose that $L$ has $k$ components, with $k \geq 2$. Because of Property 7 and the inductive hypothesis we may assume that $L$ is not split. Let $T$ be a tree having root $P$ and only one changing branch, where the crossings that have been changed are those that must be changed in order to "lift" the first component of $L$ above all the rest. Again, label the outermost projections of $T, P_{1}$ through $P_{n}$, with $P_{n}$ at the end of the changing branch. Hence $P_{n}$ is split and therefore equivalent to a projection having fewer crossings.

Furthermore each $P_{i}$, for $i \leq n-1$, has $m-1$ crossings and $k-1$ components. So we have,

$$
\begin{aligned}
Q_{P} & =a_{n} z^{1-k} Q_{P_{n}}^{\prime}+\sum a_{i}\left(-z / b_{l}\right) z^{2-k} Q_{P_{i}}^{\prime} \\
& =z^{1-k}\left[a_{n} Q_{P_{n}}^{\prime}-z^{2} \sum a_{i} b_{i}^{-1} Q_{P_{l}}^{\prime}\right] .
\end{aligned}
$$

Thus $Q_{P}^{\prime}$ has only non-negative even powers of $z$ and furthermore $Q_{P}^{\prime}(x, y, 0)=a_{n} Q_{P_{n}}^{\prime}(x, y, 0) \neq 0$.

We can provide examples of distinct knots having the same polynomials by means of mutations. Recall that a mutation of a projection $P$ is obtained as follows. Suppose $D$ is a disk in $\mathbf{R}^{2}$ that meets $P$ transversely in 4 points. Let $P^{\prime}=P \cap D$. We may alter $P$ by removing $P^{\prime}$ and then replacing it via some rigid motion of $D$ which leaves $P^{\prime} \cap \partial D$ invariant. Such an alteration is called a mutation. There are 8 possible ways to replace $P^{\prime}$ with each corresponding to an element of the dihedral group $D_{4}$. However, since $P$ is oriented, some rule by which the new projection is oriented must be adopted. For some of the possibilities no such obvious rule exists. Therefore consider only the four mutations illustrated in Figure 3.2. (We may also compose these to obtain flips about the horizontal axis). 


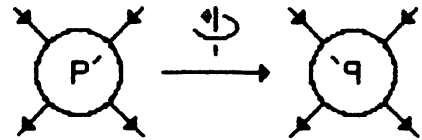

๑) กip

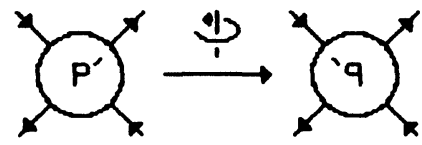

c) nip and reverse orientations of components entirely inside $D$

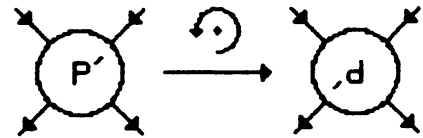

b) rotate and reverse orientations of components entirely inside $D$

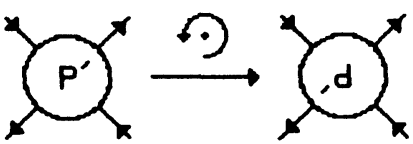

d) rotote

FigURE 3.2

11. Suppose $P$ is any projection and that $m P$ is obtained from it by one of the mutations shown in Figure 3.2. Then $Q_{P}=Q_{m P}$.

Proof. Suppose $c$ is a crossing in $P$ and that $m c$ is the crossing in $m P$ which $c$ is sent to under the mutation. Then in each of the four possible mutations $c$ and $m c$ are either both right handed or both left handed. (In cases (b) and (c) we reversed the orientation of components entirely inside $D$ so that this would be true.) Hence it follows that the operations of mutating $P$ and changing or smoothing $c$ commute.

Now let $R$ be a resolution of $P$ such that each outermost projection of $R$ is descending with respect to some choice of ordering and pointing where the distinguished points lie outside of $D$ if possible. Let $m R$ be obtained from $R$ by mutating each projection of $R$ in the same way. Now since mutation commutes with changing or smoothing a crossing it follows that $m R$ is indeed a tree of projections with $m P$ as root. Furthermore,
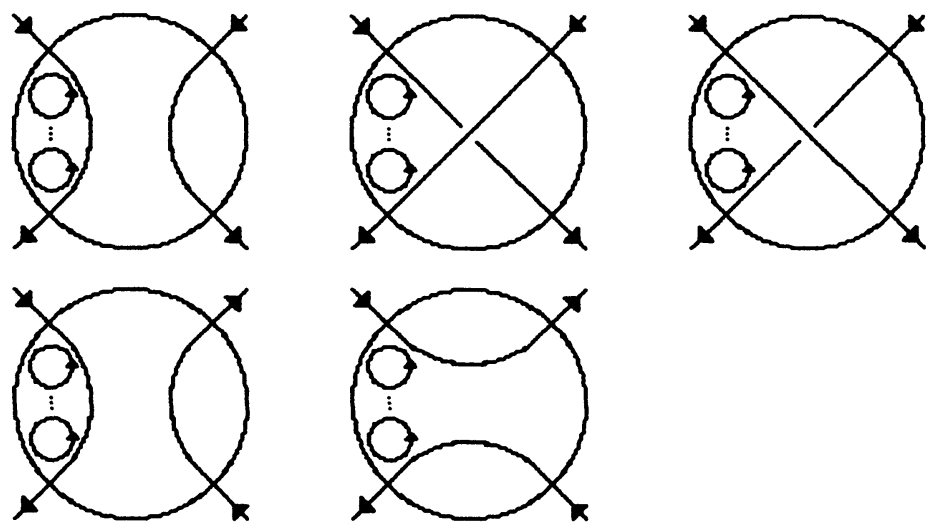

FIGURE 3.3 
$m R$ is a resolution of $m P$. For suppose $P^{\prime \prime}$ is an outermost projection of $R$. Then inside $D, P^{\prime \prime}$ can be isotoped, rel $\partial D$, to one of the projections shown in Figure 3.3. Hence $m P^{\prime \prime}$ is isotopic to $P^{\prime \prime}$ and thus is an unlink of the same number of components.

Finally, since the mutation preserved the right or left handedness of every crossing, $m R$ will produce the same polynomial as $R$.

It is easy to exhibit different knots or links which are related by a sequence of mutations and hence have the same polynomials. For example, consider the pretzel knot or link shown in Figure 3.4 where $r_{i}$ is the number of right handed half twists in the $i$ th band. Then the $r_{i}$ 's may be permuted by means of mutations. Hence, examples of distinct pretzel knots or links with identical polynomials can be produced in this way.

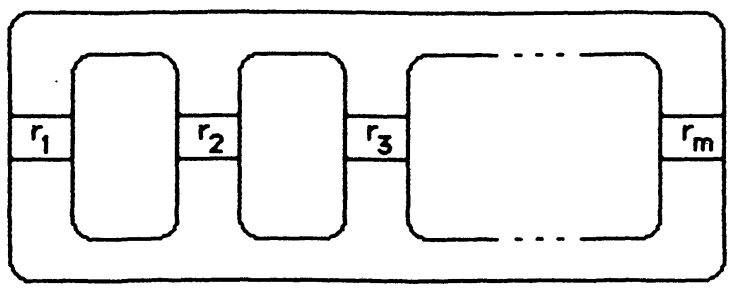

FIgURE 3.4

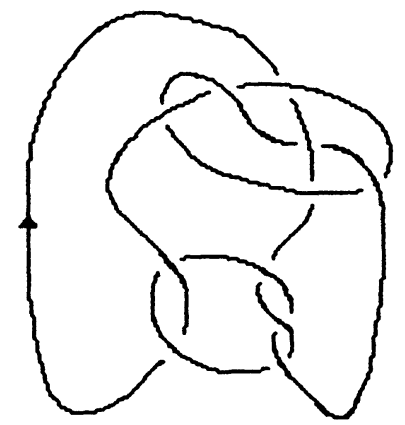

Conway

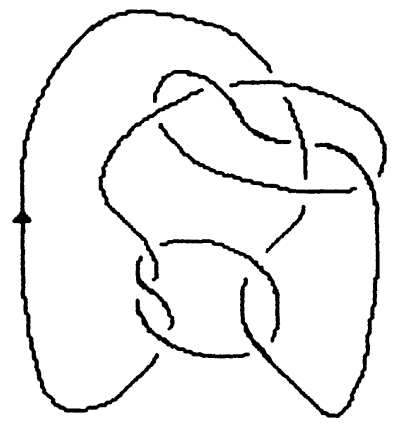

Kinoshito-Terasaka

$$
\begin{aligned}
Q=x^{-3} y^{-4} & {\left[2 x^{2} y^{5}+7 x^{3} y^{4}+6 x^{4} y^{3}+2 x^{5} y^{2}\right.} \\
- & \left(3 x y^{4}+11 x^{2} y^{3}+11 x^{3} y^{2}+3 x^{4} y\right) z^{2} \\
+ & \left.\left(6 x y^{2}+6 x^{2} y+x^{3}+y^{3}\right) z^{4}-(x+y) z^{6}\right]
\end{aligned}
$$


As a further example, consider the Conway and Kinoshita-Terasaka knots shown in Figure 3.5. They are related by the obvious mutation, and hence, have the same polynomial, which is given in the figure. The polynomial is nontrivial, yet reduces to a trivial Conway polynomial. These knots can be shown to be different by examining representations of their fundamental groups [10].

On the other hand, the square and granny knots shown in Figure 3.6 have isomorphic fundamental groups and hence the same Alexander invariant, yet different polynomials. (We may compute their polynomials by first computing the polynomial of the right handed trefoil, using Property 4 to obtain $Q$ for the left handed trefoil, and then Property 8.) Clearly then, $Q_{L}$ depends on more than the fundamental group or the Alexander invariant.

As a final example, consider the two links shown in Figure 3.7. They have homeomorphic complements but different polynomials. So $Q_{L}$ is not determined by the complement of $L$. But in this case neither is $\nabla_{L}$, although this cannot be the case for knots where the complement determines the Alexander invariant and hence $\nabla_{L}$.

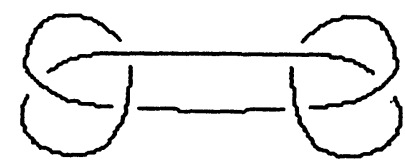

$K=$ square knot

$Q_{K}=x^{-2} y^{-2}\left(-2 x y-y^{2}+z^{2}\right)\left(-2 x y-x^{2}+z^{2}\right)$

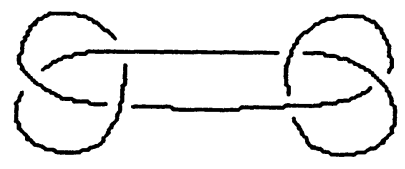

$J=$ granny knot

$Q_{J}=y^{-4}\left(-2 x y-x^{2}+z^{2}\right)^{2}$

Figure 3.6

$L_{1}$

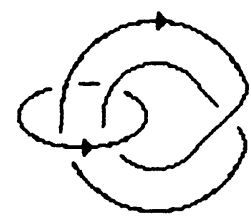

$L_{2}$

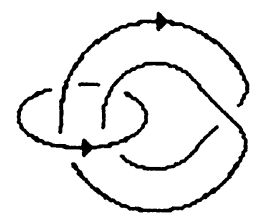

$$
\begin{aligned}
& Q_{L_{1}}=x^{-3} z^{-1}\left(-x^{2} y^{2}-x y^{3}+3 x y z^{2}+y^{2} z^{2}-z^{4}\right) \\
& Q_{L_{2}}=x^{-2} z^{-1}\left(-x y^{2}-y^{3}+y z^{2}-x z^{2}\right)
\end{aligned}
$$




\section{REFERENCES}

[1] J. H. Conway, An Enumeration of Knots and Links, and Some of Their Algebraic Properties, Computational Problems in Abstract Algebra (John Leech, ed.), Pergamon Press, Oxford and New York, 1969.

[2] P. Freyd and D. Yetter, A new invariant for knots and links, preprint.

[3] P. Freyd, J. Hoste, W. B. R. Lickorish, K. Millett, A Ocneau, and D. Yetter, A new polynomial invariant of knots and links, Bull. Amer. Math. Soc., 12 (1985), 239-246.

[4] C. A. Giller, A Family of links and the Conway calculus, Trans. Amer. Math. Soc., 270 (1982), 75-109.

[5] V. Jones, A polynomial invariant for knots via non Neumann algebras, Bull. Amer. Math. Soc., V12 No. 1 (1985), 103-111.

[6] L. Kauffman, The Conway polynomial, Topology, 20 (1981), 101-108.

[7] W. B. R. Lickorish and K. Millett, A polynomial invariant of oriented links, preprint.

[8] A. Ocneanu, A polynomial invariant for knots: A combinatorial and an algebraic approach, preprint.

[9] J. Przytycki and P. Traczyk, Invariants of links of Conway type, preprint.

[10] R. Riley, Homomorphisms of knot groups on finite groups, Math. Comp., 25 (1971), 603-619.

Received April 1, 1985.

RUTGERS UNIVERSITY

NEW BRUNSWICK, NJ 08903 


\section{PACIFIC JOURNAL OF MATHEMATICS EDITORS}

\author{
V. S. VARADARAJAN \\ (Managing Editor) \\ University of California \\ Los Angeles, CA 90024 \\ Herbert Clemens \\ University of Utah \\ Salt Lake City, UT 84112 \\ R. FINN \\ Stanford University \\ Stanford, CA 94305
}

\author{
HERMANN FLASCHKA \\ University of Arizona \\ Tucson, AZ 85721 \\ RAMESH A. GANGOLLI \\ University of Washington \\ Seattle, WA 98195 \\ VAUGHAN F. R. JONES \\ University of California \\ Berkeley, CA 94720 \\ ROBION KIRBY \\ University of California \\ Berkeley, CA 94720
}

C. C. MOORE

University of California

Berkeley, CA 94720

H. SAMELSON

Stanford University

Stanford, CA 94305

HAROLD STARK

University of California, San Diego La Jolla, CA 92093

\section{ASSOCIATE EDITORS}
R. ARENS
E. F. BECKENBACH (1906-1982)
B. H. NEUMANN
F. WOLF
K. YosHIDA

\section{SUPPORTING INSTITUTIONS}

\begin{abstract}
UNIVERSITY OF ARIZONA
UNIVERSITY OF BRITISH COLUMBIA

CALIFORNIA INSTITUTE OF TECHNOLOGY

UNIVERSITY OF CALIFORNIA

MONTANA STATE UNIVERSITY

UNIVERSITY OF NEVADA, RENO

NEW MEXICO STATE UNIVERSITY OREGON STATE UNIVERSITY
\end{abstract}

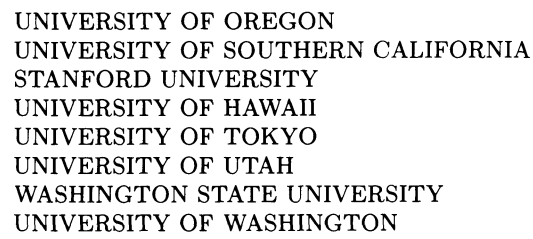

The Supporting Institutions listed above contribute to the cost of publication of this Journal, but they are not owners or publishers and have no responsibility for its content or policies.

Mathematical papers intended for publication in the Pacific Journal of Mathematics should be in typed form or offset-reproduced (not dittoed), double spaced with large margins. Please do not use built up fractions in the text of the manuscript. However, you may use them in the displayed equations. Underline Greek letters in red, German in green, and script in blue. The first paragraph must be capable of being used separately as a synopsis of the entire paper. In particular it should contain no bibliographic references. Please propose a heading for the odd numbered pages of less than 35 characters. Manuscripts, in triplicate, may be sent to any one of the editors. Please classify according to the scheme of Math. Reviews, Index to Vol. 39. Supply name and address of author to whom proofs should be sent. All other communications should be addressed to the managing editor, or Elaine Barth, University of California, Los Angeles, California 90024.

There are page-charges associated with articles appearing in the Pacific Journal of Mathematics. These charges are expected to be paid by the author's University, Government Agency or Company. If the author or authors do not have access to such Institutional support these charges are waived. Single authors will receive 50 free reprints; joint authors will receive a total of 100 free reprints. Additional copies may be obtained at cost in multiples of 50 .

The Pacific Journal of Mathematics is issued monthly as of January 1966. Regular subscription rate: $\$ 190.00$ a year (5 Vols., 10 issues). Special rate: $\$ 95.00$ a year to individual members of supporting institutions.

Subscriptions, orders for numbers issued in the last three calendar years, and changes of address should be sent to Pacific Journal of Mathematics, P.O. Box 969, Carmel Valley, CA 93924, U.S.A. Old back numbers obtainable from Kraus Periodicals Co., Route 100, Millwood, NY 10546.

The Pacific Journal of Mathematics at P.O. Box 969, Carmel Valley, CA 93924 (ISSN 0030-8730) publishes 5 volumes per year. Application to mail at Second-class postage rates is pending at Carmel Valley, California, and additional mailing offices. Postmaster: send address changes to Pacific Journal of Mathematics, P.O. Box 969, Carmel Valley, CA 93924.

PUBLISHED BY PACIFIC JOURNAL OF MATHEMATICS, A NON-PROFIT CORPORATION Copyright (C) 1986 by Pacific Journal of Mathematics 


\section{Pacific Journal of Mathematics}

Vol. 124, No. $2 \quad$ June, 1986

Philip Lee Bowers, Nonshrinkable "cell-like" decompositions of $s \ldots \ldots .257$

Aurelio Carboni and Ross Street, Order ideals in categories .......... 275

Leoni Dalla, Increasing paths on the one-skeleton of a convex compact set in

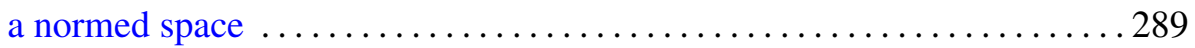

Jim Hoste, A polynomial invariant of knots and links ............... 295

Sheldon Katz, Tangents to a multiple plane curve ................... 321

Thomas George Lucas, Some results on Prüfer rings $\ldots \ldots \ldots \ldots \ldots \ldots 33$

Pham Anh Minh, Modular invariant theory and cohomology algebras of

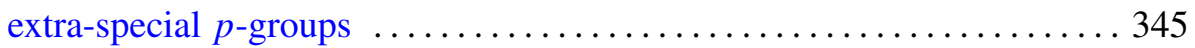

Ikuko Miyamoto, On inclusion relations for absolute Nörlund

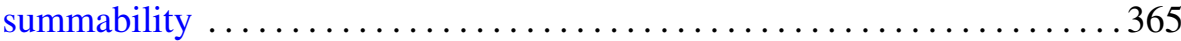

A. Papadopoulos, Geometric intersection functions and Hamiltonian flows on the space of measured foliations on a surface ............. 375

Richard Dean Resco, J. Toby Stafford and Robert Breckenridge

Warfield, Jr., Fully bounded $G$-rings $\ldots \ldots \ldots \ldots \ldots \ldots \ldots \ldots \ldots 4$

Haskell Paul Rosenthal, Functional Hilbertian sums .................417

Luen-Fai Tam, Regularity of capillary surfaces over domains with corners: borderline case ................................. 469

Hugh C. Williams, The spacing of the minima in certain cubic lattices .....483 